\section{International Scientific Journal Theoretical \& Applied Science}

\author{
p-ISSN: 2308-4944 (print) e-ISSN: 2409-0085 (online) \\ Year: 2015 Issue: 08 Volume: 28 \\ Published: $30.08 .2015 \quad$ http://T-Science.org
}

SECTION 7. Mechanics and machine construction.
Denis Alexandrovich Chemezov Master of Engineering and Technology, Corresponding member of International Academy of Theoretical and Applied Sciences, Lecturer of Vladimir Industrial College, Russia chemezov-da@yandex.ru

\title{
ASSESSMENT OF PLASTIC STRAIN OF WORKPIECE MATERIAL DURING COLD DRAWING
}

Abstract: The article presents the analysis of the distribution of plastic strain in the workpiece, made of aluminum alloy, during drawing. Defined values of the stresses and strains of the material in the zone of intensive treatment of pressure.

Key words: drawing, plastic strain, workpiece.

Language: Russian

Citation: Chemezov DA (2015) ASSESSMENT OF PLASTIC STRAIN OF WORKPIECE MATERIAL DURING COLD DRAWING. ISJ Theoretical \& Applied Science 08 (28): 9-13.

Soi: http://s-o-i.org/1.1/TAS-08-28-2 Doi: crossef http://dx.doi.org/10.15863/TAS.2015.08.28.2

\section{ОЦЕНКА ПЛАСТИЧЕСКОЙ ДЕФОРМАЦИИ МАТЕРИАЛА ЗАГОТОВКИ ПРИ ХОЛОДНОМ ВОЛОЧЕНИИ}

Аннотация: В статье представлен анализ распределения пластической деформации в заготовке, изготовленной из алюминиевого сплава, при волочении. Определены величины напряжений и деформаций материала в зоне интенсивной обработки давлением.

Ключевые слова: волочение, пластическая деформация, заготовка.

Обработка металлических сплавов давлением имеет ряд преимуществ по сравнению с лезвийной обработкой резанием:

1) безотходное или малоотходное производство, т. е. получение зачастую готового изделия при минимальном расчетном коэффициенте использования материала заготовки;

2) упрочнение поверхностного слоя (наклеп) материала обеспечивающее увеличение износостойкости изделия во время эксплуатации;

3) в ряде случаев изменение структуры материала заготовки.

Алюминиевые сплавы хорошо поддаются обработке давлением: прокатке, ковке, прессованию и др. Качественный анализ напряженно-деформированного состояния заготовок, из алюминиевого сплава, подвергаемых равноканальному угловому прессованию по различным схемам представлен в работах $[1,2]$, прокатки в работе [3], листовой штамповки в работе [4].

Одним из способов обработки металлических сплавов давлением является волочение, представляющее собой протягивание прутков различных профилей и труб через специальный инструмент (волока) со ступенчатым отверстием, сечение которого меньше сечения заготовки [5]. Многопроходным протягиванием можно получить проволоку наружным диаметром менее 1 мм.

Процесс волочения сопровождается интенсивным удлинением деформируемой заготовки. Материал заготовки в зоне формообразования подвергается одновременно и растяжению и сжатию. Пластически деформированный материал будет обладать уже другими характеристиками. Исследование процесса волочения методом имитационного моделирования позволит получить достоверную оценку напряженно-деформированного состояния материала заготовки.

Расчет задачи механики деформируемой заготовки методом конечных элементов выполнялся в программной среде LS-DYNA.

Волока представляет собой втулку. Ступенчатое отверстие состоит из следующих участков: заборная/деформирующая зона 


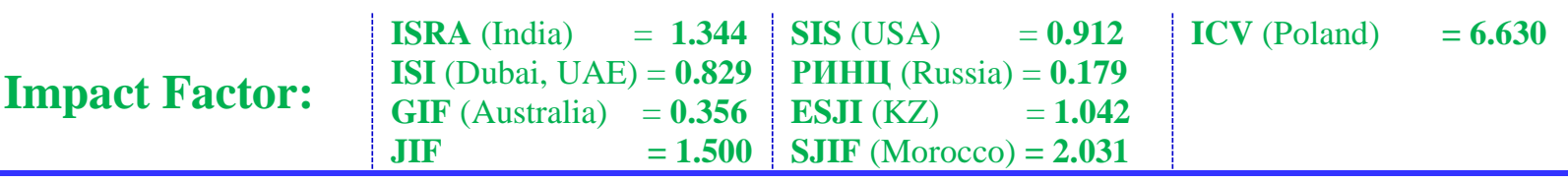

конической формы $\left(18^{\circ}\right.$ в соответствии с конструкцией волочильного инструмента), калибрующий пояс цилиндрической формы $\left(\varnothing 16.5\right.$ мм) и выходной конус $\left(70^{\circ}\right)$ [6]. Конструкция волоки и ее габаритные размеры представлены на рис. 1.

В качестве деформируемой заготовки принят пруток круглого поперечного сечения с наружным диаметром 20 мм. Материал заготовки - алюминиевый сплав со следующими физико- механическими свойствами [7]: плотность - 2770 кг/м³ модуль Юнга - $71000 \mathrm{MПа;} \mathrm{коэффициент}$ Пуассона - 0.33; предел текучести на растяжение - 280 МПа; модуль сдвига - 500 МПа. Для задания усилия и направления волочения применяется специальное захватное тянущее устройство [8]. Захват производится клещами за проточенный наружный диаметр прутка (Ø15 мм) со стороны выходного конуса волоки.

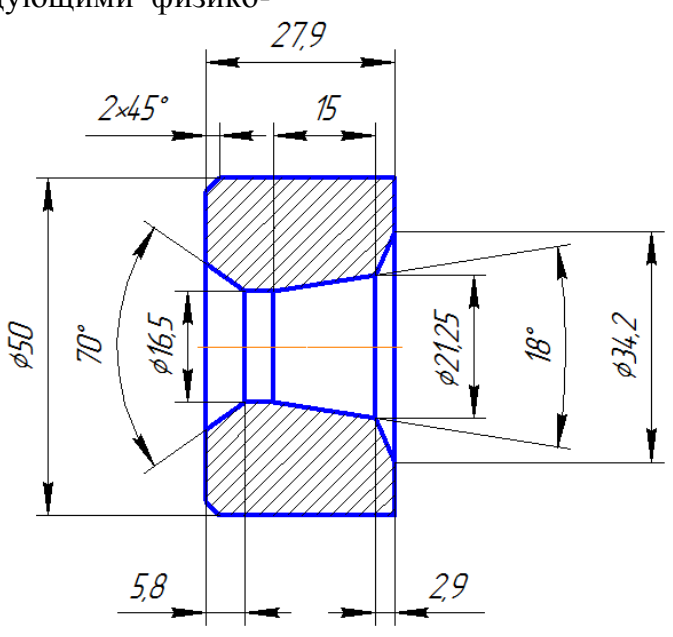

Рисунок 1 - Чертеж волоки.

Построенные трехмерные твердотельные модели волоки и прутка были разбиты на конечные элементы [9] в виде сетки (рис. 2).
Общее количество элементов составляло 36448 (модель волоки - 6647, модель прутка - 29801). Размер одного элемента - 2 мм.

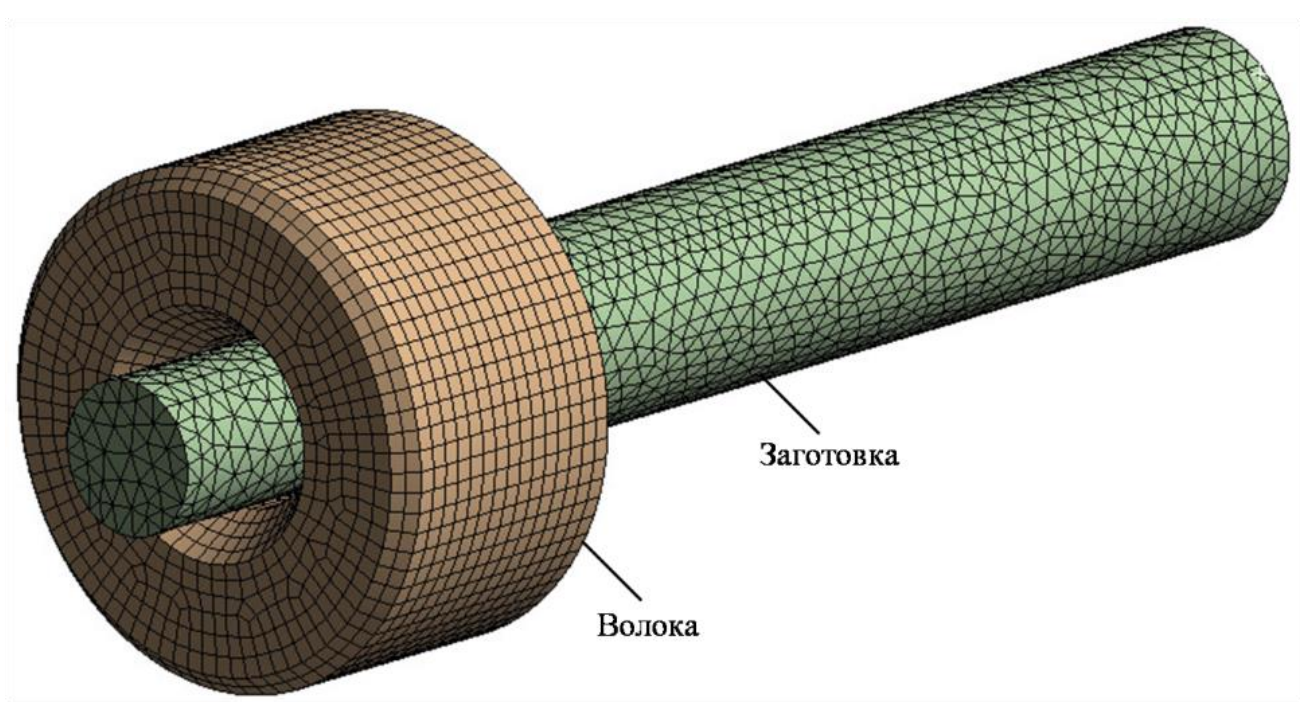

Рисунок 2 - Разбиение твердотельных моделей волоки и заготовки на конечные элементы.

Установка параметров и граничные условия моделирования (расчета) процесса волочения представлены в табл. 1. и на рис. 3 соответственно. 


\begin{tabular}{|c|c|c|c|c|c|}
\hline Impact Factor: & $\begin{array}{l}\text { ISRA (India) } \\
\text { ISI (Dubai, UAF } \\
\text { GIF (Australia) } \\
\text { JIF }\end{array}$ & $\begin{array}{l}=1.344 \\
=0.829 \\
=0.356 \\
=1.500\end{array}$ & $\begin{array}{l}\text { SIS (USA) } \\
\text { PИНЦ (Russia } \\
\text { ESJI (KZ) } \\
\text { SJIF (Morocco }\end{array}$ & $\begin{array}{l}=0.912 \\
=0.179 \\
=1.042 \\
=2.031\end{array}$ & ICV (Poland) \\
\hline
\end{tabular}

Параметры моделирования динамики процесса волочения.

Таблица 1

\begin{tabular}{|c|c|}
\hline Параметр & Значение параметра \\
\hline Максимальное количество циклов & 100000 \\
\hline Время окончания (конечное) & $7 \mathrm{c}$ \\
\hline Максимальная ошибка энергии & $10 \%$ \\
\hline Количество оболочковых подслоев & 3 \\
\hline Поправочный коэффициент сдвига оболочки & 0.8333 \\
\hline
\end{tabular}

Модель волоки принята жестким телом, т. е. все поверхности не деформировались (выделены фиолетовым цветом с меткой В). Метка С на торцевой поверхности характеризует неподвижность модели волоки относительно модели заготовки. Стрелкой (метка А) показано направление усилия волочения (25 кН). Модель заготовки принята пластическим материалом. Заготовка перемещалась только по координатной оси X (метка D). Заготовка обрабатывалась в холодном состоянии.

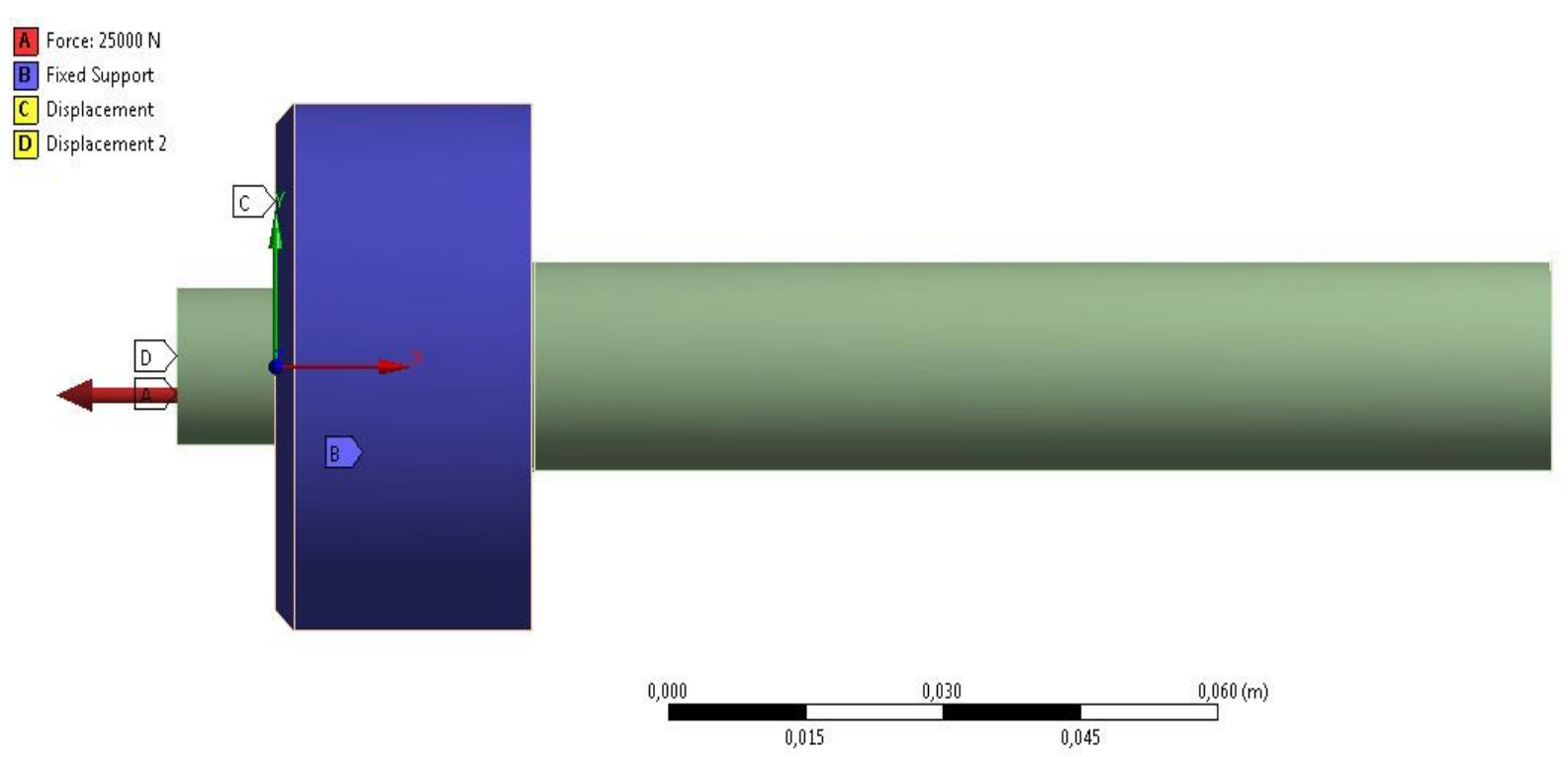

Рисунок 3 - Граничные условия для моделирования динамики процесса волочения. Координатная система сгенерирована в модуле Explicit Dynamic программной среды Ansys Workbench.

Результаты реализации моделирования процесса волочения представлены в виде расчетных значений параметров (табл. 2) и интерактивных эпюр (рис. 4) характеризующих напряженно-деформированное состояние

Таблица 2

Параметры, характеризующие напряженно-деформированное состояние заготовки.

\begin{tabular}{|c|c|c|}
\hline \multirow{2}{*}{ Параметр } & \multicolumn{2}{|c|}{ Величина } \\
\hline & Минимальная & Максимальная \\
\hline Напряжение по оси X, H/мм² & -514.5 & 735.5 \\
\hline Напряжение по оси Y, H/мм² & -951.9 & 473.9 \\
\hline Напряжение по оси Z, H/мм² & -950.7 & 510.6 \\
\hline Эффективное напряжение (напряжение по фон Мизесу), Н/мм² & 0 & 620.6 \\
\hline Максимальное напряжение сдвига, H/мм² & 0 & 347.4 \\
\hline Максимальное главное напряжение (1-ое), Н/мм² & -494.1 & 804.1 \\
\hline Второе главное напряжение, $\mathrm{H} / \mathrm{Mм}^{2}$ & -825.5 & 513.7 \\
\hline Минимальное главное напряжение (3-ье), Н/мм² & -1029.4 & 245.4 \\
\hline Перемещение по оси X, мм & -25.9 & 0 \\
\hline Перемещение по оси Y, мм & -2.3 & 2.2 \\
\hline
\end{tabular}


Impact Factor:

\begin{tabular}{|c|c|}
\hline ISRA (India) & SIS (USA) \\
\hline $\mathrm{i}, \mathrm{UAE})=\mathbf{0 . 8}$ & РИНЦ (Russia) = \\
\hline & ESJI $(\mathrm{KZ}) \quad=\mathbf{1 . 0 4 2}$ \\
\hline$=1.5$ & SJIF $($ Morocco $)=\mathbf{2 . 0 3}$ \\
\hline
\end{tabular}

\begin{tabular}{|c|c|c|}
\hline Перемещение по оси Z, мм & -2.1 & 2.2 \\
\hline Результирующее перемещение, мм & 0 & 25.9 \\
\hline Скорость деформации по оси X, мм/мс & -88.2 & 8.8 \\
\hline Скорость деформации по оси Ү, мм/мс & -45.2 & 28.2 \\
\hline Скорость деформации по оси Z, мм/мс & -24.9 & 30.3 \\
\hline Результирующая скорость деформации, мм/мс & 0 & 89.2 \\
\hline
\end{tabular}

Напряженное состояние материала заготовки было определено по характеру деформаций возникающих по 3 координатным осям (см. рис. 4, а). По координатной оси $\mathrm{X}$ преобладает растяжение материала $\left(735.5 \mathrm{H} / \mathrm{Mm}^{2}\right)$, по координатным осям Y и Z - сжатие материала

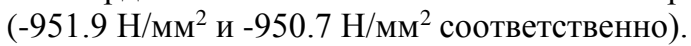

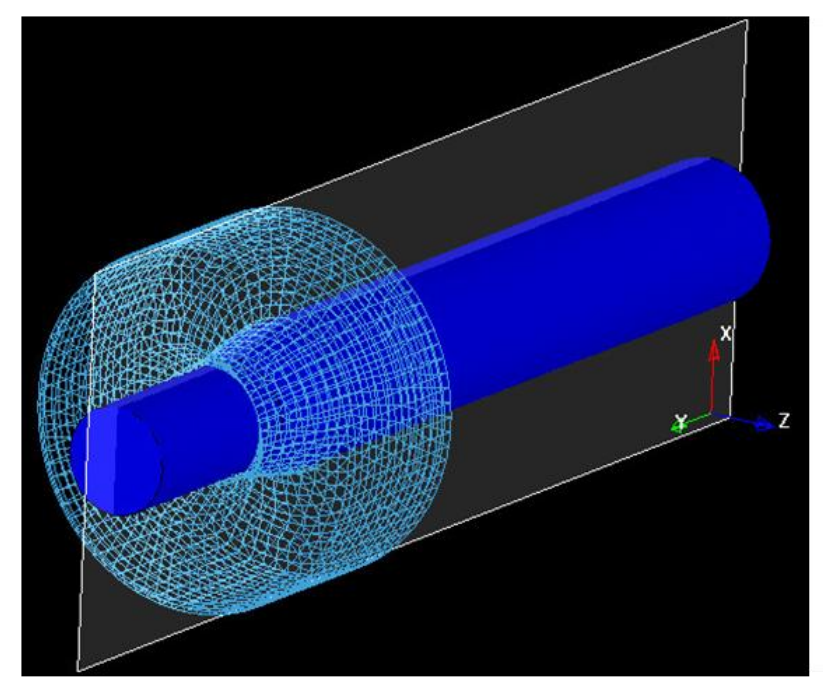

a)

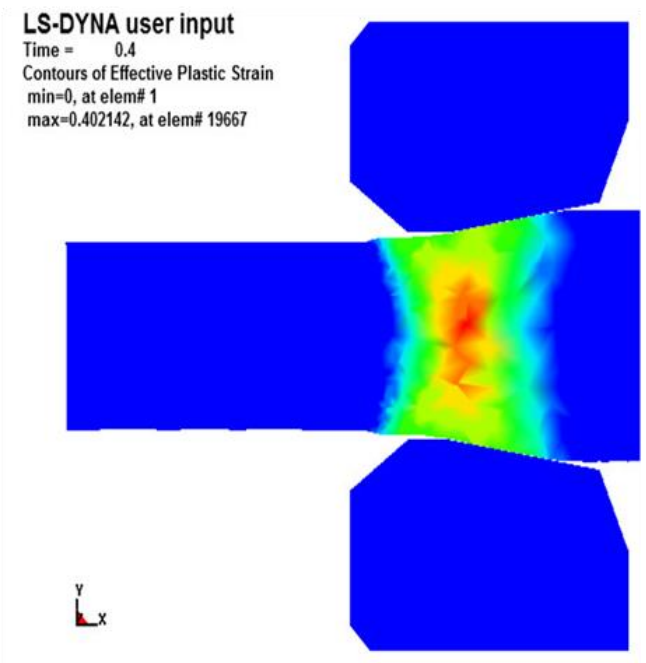

в)
Продольное перемещение заготовки на 26 мм, относительно заборной зоны волочильного инструмента, способствует увеличению ее общей длины на 10\% (при заданных режимах обработки и геометрических размерах волоки). Полная энергия (сумма кинетической и внутренней энергий) затрачиваемая на процесс волочения заготовки составила 60 кДж.

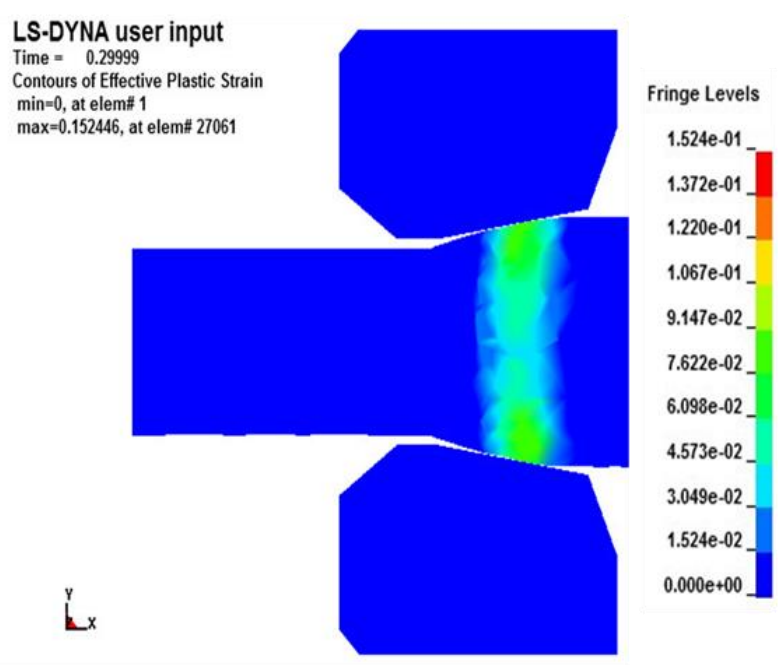

б)

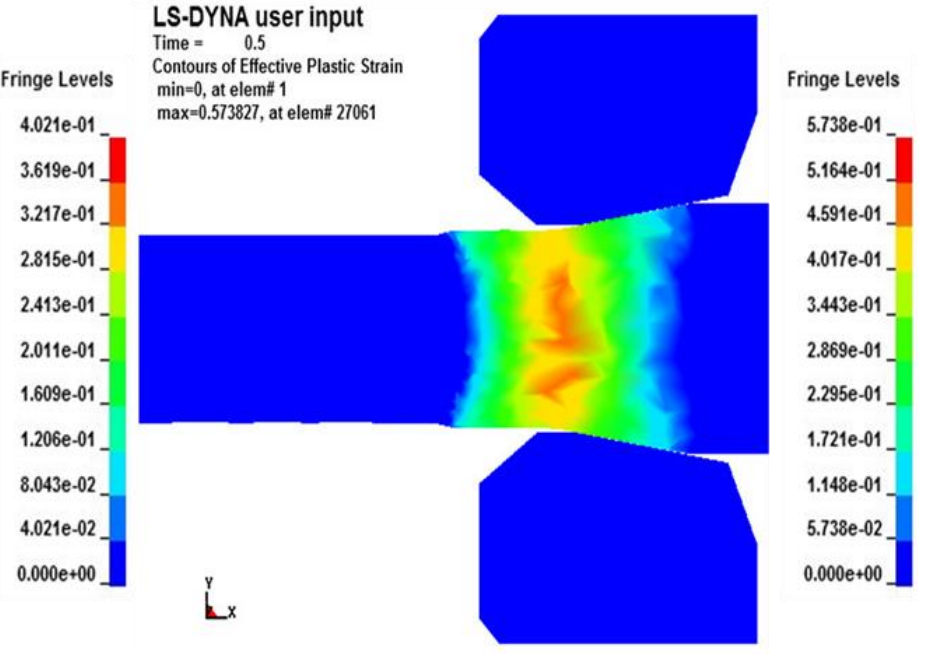

г)

Рисунок 4 - Результаты моделирования процесса волочения: а - сечение моделей плоскостью ХY; б, В, Г - эпюры эффективной пластической деформации заготовки по мере протягивания заготовки через отверстие волоки.

Наибольшая пластическая деформация материала заготовки происходит в месте перехода малого диаметра конуса деформирующей зоны в цилиндрический

ISPC Science and Education, 
калибрующий пояс волоки (в продольном и поперечном сечениях). Слои материала, расположенные ближе к осевой линии заготовки, деформируются больше чем поверхностные слои.

Величина пластической деформации поверхностного слоя (глубиной до 2 мм) материала заготовки в деформирующей зоне волоки находится из зависимости (1)

$$
\varepsilon_{p l}=\frac{d-0.135 L-d_{v a r}}{0.12 d},
$$

где $\varepsilon_{p l}$ - коэффициент пластической деформации материала заготовки; $d$ - наружный диаметр заготовки до обработки давлением, мм; $L$ - длина деформирующей зоны волоки на которой происходит фактическая обработка материала заготовки, $L=k l_{n}$ ( $l_{n}-$ шаг разбиения длины деформирующей зоны волоки на равное количество участков, $k$ - количество участков), мм; $d_{v a r}-$ наружный диаметр (переменный) заготовки непосредственно подвергающийся обработке в деформирующей зоне волоки, мм.

На степень пластической деформации материала заготовки будут существенно влиять длина и угол при вершине деформирующей части волоки. При соотношении длины $L>1.5 d$ и угла при вершине $2 \alpha>25^{\circ}$ деформирующей зоны волочильного инструмента происходит увеличение площади контактных поверхностей заготовки с формообразующим отверстием. Это приводит к дополнительным энергозатратам (увеличение усилия волочения) и значительным пластическим деформациям материала заготовки.

\section{References:}

1. Chemezov DA (2015) Comparative analysis of stress-strain state of workpieces made of aluminium alloy during of the equal channel angular pressing process according to different schemes. ISJ Theoretical \& Applied Science 07 (27): 52-64. Soi: http://s-o-i.org/1.1/TAS-07$\underline{27-10}$ Doi http://dx.doi.org/10.15863/TAS.2015.07.27.10

2. Chemezov DA (2015) Modelling and analysis of process of equal-channel angular pressing of metal alloys. New University. Technical sciences. 2015. 5-6 (39-40). - pp. 42 - 52. ISSN 2221 - 9552. DOI: 10.15350/22219552.2015.5-6

3. Maksimov EA (2014) Research of contact friction forces and specific pressures in the time of rolling of strips, made of aluminium and its alloys. Ore \& Metals. 2014. 6 (858). - pp. 8789.

4. Galkin VV, Kalinin AB, Pachurin GV, Ermakov DY (2014) Limiting degree dome sheet product with spherical bottom in hot strain. Fundamental research. 2014. 5-5. - pp. 939-944.

5. (2015) Volochenie. Available: https://ru.wikipedia.org/wiki/Волочение (Accessed: 31.07.2015).

6. (2015) Volochenie metalla. Volochenie provoloki. Tehnologiya volocheniya. Oborudovanie dlya volocheniya. Available: http://www.mtomd.info/archives/1999 (Accessed: 31.07.2015).

7. (2015) Aluminiy i ego splavy. Available: http://www.1 metal.ru/info-alsplav.html (Accessed: 31.07.2015).

8. (2015) Volochilnye stany. Oborudovanie dlya volochilnyh tsehov. Volochenie provoloki. Available: $\quad$ http://www.rollingmills.ru/drawing machines.php (Accessed: 31.07.2015).

9. (2015) Method konechnyh elementov Available:

https://ru.wikipedia.org/wiki/Метод_конечных элементов (Accessed: 31.07.2015). 